\title{
Fixed Point Theorems on Ordered Metric Spaces through a Rational Contraction
}

\author{
Poom Kumam, ${ }^{1}$ Fayyaz Rouzkard, ${ }^{2}$ Mohammad Imdad, ${ }^{2}$ and Dhananjay Gopal ${ }^{3}$ \\ ${ }^{1}$ Department of Mathematics, Faculty of Science, King Mongkut's University of Technology Thonburi (KMUTT), Bang Mod, \\ Bangkok 10140, Thailand \\ ${ }^{2}$ Department of Mathematics, Aligarh Muslim University, Aligarh 202002, India \\ ${ }^{3}$ Department of Applied Mathematics \& Humanities, S. V. National Institute of Technology, Surat 395007, India
}

Correspondence should be addressed to Poom Kumam; poom.kum@kmutt.ac.th

Received 10 May 2013; Accepted 9 July 2013

Academic Editor: Salvador Hernandez

Copyright (C) 2013 Poom Kumam et al. This is an open access article distributed under the Creative Commons Attribution License, which permits unrestricted use, distribution, and reproduction in any medium, provided the original work is properly cited.

\begin{abstract}
Ran and Reurings (2004) established an interesting analogue of Banach Contraction Principle in a complete metric space equipped with a partial ordering and also utilized the same oneto discuss the existence of solutions to matrix equations. Motivated by this paper, we prove results on coincidence points for a pair of weakly increasing mappings satisfying a nonlinear contraction condition described by a rational expression on an ordered complete metric space. The uniqueness of common fixed point is also discussed. Some examples are furnished to demonstrate the validity of the hypotheses of our results. As an application, we derive an existence theorem for the solution of an integral equation.
\end{abstract}

\section{Introduction with Preliminaries}

A variety of generalizations of the Classical Banach Contraction Principle [1] are available in the existing literature of metric fixed point theory. The majority of these generalizations are obtained by improving the underlying contraction condition (e.g., [2]). Presently, there is vigorous research activity to prove existence results on complete metric spaces equipped with a partial ordering. In fact, various existence and uniqueness theorems on fixed and common fixed point for monotone mappings are of paramount importance in the study of nonlinear equations which generate natural interest to establish usable fixed point theorems in partial metric spaces (e.g., [1-24]).

Very recently, Harjani et al. [25] proved a fixed point theorem in partially ordered metric spaces satisfying a contractive condition of rational type due to Jaggi [26]. The aim of this paper is to prove some results of Harjani et al. [25] type for a pair of self-mappings. We accomplish this using the concept of weakly increasing property due to Nashine and Samet [14] (also see $[4,27,28]$ ). Some examples are also furnished to demonstrate the validity of the hypotheses of our results. As an application, we establish the existence of solution to an integral equation (also see $[2,23,29,30]$ ).

Before presenting our results, we recall some notations, definitions, and examples required in our subsequent discussions.

Definition 1 . Let $\mathscr{X}$ be a nonempty set. Then $(\mathscr{X}, d, \preceq)$ is called an ordered (partial) metric space if

(i) $(X, \preceq)$ is a partially ordered set and (ii) $(\mathscr{X}, d)$ is a metric space.

Definition 2 . Let $(\mathscr{X}, \preceq)$ be a partially ordered set. Then

(a) elements $x, y \in \mathscr{X}$ are called comparable with respect to " $\leq$ " if either $x \leq y$ or $y \leq x$;

(b) a mapping $\mathscr{T}: \mathscr{X} \rightarrow \mathscr{X}$ is called nondecreasing with respect to " $\leq$ " if $x \leq y$ implies $\mathscr{T} x \leq \mathscr{T} y$.

Let $\mathscr{X}$ be a nonempty set and $\mathscr{R}: \mathscr{X} \rightarrow \mathscr{X}$ be a given mapping. For every $x \in \mathscr{X}$, we denote by $\mathscr{R}^{-1}(x)$, the subset of $\mathscr{X}$ defined by

$$
\mathscr{R}^{-1}(x):=\{u \in \mathscr{X} \mid \mathscr{R} u=x\} .
$$


Definition 3. Let $(\mathscr{X}, \preceq)$ be a partially ordered set and let $\mathscr{T}, \mathscr{R}: \mathscr{X} \rightarrow \mathscr{X}$ be two mappings such that $\mathscr{T} \mathscr{X} \subseteq \mathscr{R} \mathscr{X}$. We say that $\mathscr{T}$ is weakly increasing with respect to $\mathscr{R}$ if and only if for all $x \in \mathscr{X}$, one has:

$$
\mathscr{T} x \preceq \mathscr{T} y, \quad \forall y \in \mathscr{R}^{-1}(\mathscr{T} x) .
$$

Remark 4. If $\mathscr{R}: \mathscr{X} \rightarrow \mathscr{X}$ is the identity mapping on $\mathscr{X}$, then $\mathscr{T}$ is weakly increasing with respect to $\mathscr{R}$ if and only if $\mathscr{T}(x) \preceq \mathscr{T} \mathscr{T}(x)$ for all $x \in \mathscr{X}$.

As mentioned earlier, the notion of weakly increasing mappings was introduced in [4] which is presently in use (e.g. $[27,28])$. In what follows, we furnish a relatively new example to demonstrate the preceding definition.

Example 5. Consider $\mathscr{X}=[0, \infty)$ endowed with the natural order $\leq$. Define two mappings $\mathscr{T}, \mathscr{R}: \mathscr{X} \rightarrow \mathscr{X}$ as

$$
\begin{aligned}
& \mathscr{T} x= \begin{cases}x^{2}+1, & 0 \leq x<1 \\
1, & 1 \leq x,\end{cases} \\
& \mathscr{R} x= \begin{cases}x^{3}+1, & 0 \leq x<1 \\
1, & 1 \leq x .\end{cases}
\end{aligned}
$$

In order to show that the mapping $\mathscr{T}$ is weakly increasing with respect to mapping $\mathscr{R}$, we distinguish three cases.

Firstly, we consider the case $x=0$. Let $y \in$ $\mathscr{R}^{-1}(\mathscr{T}(0))$, that is, $\mathscr{R}(y)=\mathscr{T}(0)=1$ so that $\mathscr{R}(y)=1$ and henceforth $y=0$ or $y \geq 1$. By the definitions of $\mathscr{T}$, we have

$$
1=\mathscr{T}(0) \leq \mathscr{T}(y)=1 .
$$

Secondly, we argue the case $x \geq 1$. Let $y \in \mathscr{R}^{-1}(\mathscr{T}(x))$, that is, $\mathscr{R}(y)=\mathscr{T}(x)=1$, which amounts to say (in view of definition $R$ ) that $y=0$ or $y \geq 1$, implying thereby

$$
1=\mathscr{T}(x) \leq \mathscr{T}(y)=1 .
$$

Finally, we need to consider the case $0<x<1$. Let $y \in$ $\mathscr{R}^{-1}(\mathscr{T}(x))$, that is, $\mathscr{R}(y)=\mathscr{T}(x)$. By the definition of $\mathscr{T}$, we have $\mathscr{T}(x)=x^{2}+1$, so that $\mathscr{R}(y)=x^{2}+1$. Now, in view of definition of $\mathscr{R}$, we have $\mathscr{R}(y)=y^{3}+1$, so that $y^{3}+1=x^{2}+1$, yielding thereby $y=(x)^{2 / 3}$. Now, we have

$$
x^{2}+1=\mathscr{T}(x) \leq \mathscr{T}(y)=\mathscr{T}\left((x)^{2 / 3}\right)=(x)^{4 / 3}+1 .
$$

Thus we have shown that $\mathscr{T}$ is weakly increasing with respect to $\mathscr{R}$.

Definition 6 . Let $(\mathscr{X}, \preceq)$ be an ordered metric space. We say that $\mathscr{X}$ is regular if and only if the following hypothesis holds: if $\left\{z_{n}\right\}$ is a nondecreasing sequence in $\mathscr{X}$ with respect to $\preceq$ such that $\lim _{n \rightarrow \infty} z_{n}=z \in \mathscr{X}$, then $z_{n} \preceq z$ for all $n \in \mathbb{N}$.

Definition 7. A pair $(\mathscr{R}, \mathscr{T})$ of self-mappings of a metric space $(\mathscr{X}, d)$ is said to be compatible if and only if $\lim _{n \rightarrow+\infty} d\left(\mathscr{T} \mathscr{R} x_{n}, \mathscr{R} \mathscr{T} x_{n}\right)=0$, whenever $\left\{x_{n}\right\}$ is a sequence in $\mathscr{X}$ such that $\lim _{n \rightarrow+\infty} \mathscr{R} x_{n}=\lim _{n \rightarrow+\infty} \mathscr{T} x_{n}=z \in \mathscr{X}$.
Definition 8 (see [18]). A pair $(\mathscr{R}, \mathscr{T})$ of self-mappings of a metric space $(\mathscr{X}, d)$ is said to be reciprocally continuous if and only if $\lim _{n \rightarrow \infty} \mathscr{R} \mathscr{T} x_{n}=\mathscr{R} z$ and $\lim _{n \rightarrow \infty} \mathscr{T} \mathscr{R} x_{n}=\mathscr{T} z$ for every sequence $\left\{x_{n}\right\}$ in $\mathscr{X}$ satisfying

$$
\lim _{n \rightarrow \infty} \mathscr{R} x_{n}=\lim _{n \rightarrow \infty} \mathscr{T} x_{n}=z
$$

for some $z \in \mathscr{X}$.

Notice that a pair of continuous mappings is always reciprocally continuous but not conversely as substantiated by examples in [18].

Definition 9 (see [19]). A pair $(\mathscr{R}, \mathscr{T})$ of self mappings of a metric space $(\mathscr{X}, d)$ is said to be weakly reciprocally continuous if and only if $\lim _{n \rightarrow \infty} \mathscr{R} \mathscr{T} x_{n}=\mathscr{R} z$, for every sequence $\left\{x_{n}\right\}$ in $\mathscr{X}$ satisfying

$$
\lim _{n \rightarrow \infty} \mathscr{R} x_{n}=\lim _{n \rightarrow \infty} \mathscr{T} x_{n}=z
$$

for some $z \in \mathscr{X}$.

Evidently, every pair of reciprocally continuous mappings is always weakly reciprocally but not conversely as demonstrated in Pant et al. [19].

\section{Results}

The main result of this paper runs as follows

Theorem 10. Let $(\mathscr{X}, \preceq)$ be a partially ordered set equipped with a metric $d$ on $\mathscr{X}$ such that $(\mathscr{X}, d)$ is a complete metric space. Let $\mathscr{T}, \mathscr{R}: \mathscr{X} \rightarrow \mathscr{X}$ be two mappings satisfying (for pairs $(x, y) \in \mathscr{X} \times \mathscr{X}$ wherein $\mathscr{R} x$ and $\mathscr{R} y$ are comparable)

$$
\begin{aligned}
d(\mathscr{T} x, \mathscr{T} y) \leq & \alpha \frac{d(\mathscr{R} x, \mathscr{T} x) \cdot d(\mathscr{R} y, \mathscr{T} y)}{1+d(\mathscr{R} x, \mathscr{R} y)} \\
& +\beta d(\mathscr{R} x, \mathscr{R} y),
\end{aligned}
$$

where $\alpha, \beta$ are nonnegative real numbers with $\alpha+\beta<1$. Suppose that

(a) $\mathscr{X}$ is regular and $\mathscr{T}$ is weakly increasing with $\mathscr{R}$,

(b) the pair $(\mathscr{R}, \mathscr{T})$ is commuting as well as weakly reciprocally continuous.

Then $\mathscr{T}$ and $\mathscr{R}$ have a coincidence point. That is, there exists $u \in \mathscr{X}$ such that $\mathscr{R} u=\mathscr{T} u$.

Proof. Let $x_{0}$ be an arbitrary point in $\mathscr{X}$. Since $\mathscr{T} \mathscr{X} \subseteq \mathscr{R} \mathscr{X}$, one can inductively construct a sequence $\left\{x_{n}\right\}$ in $\mathscr{X}$ defined by

$$
\mathscr{R} x_{n}=\mathscr{T} x_{n-1}, \quad \forall n \in \mathbb{N} .
$$

As $x_{1} \in \mathscr{R}^{-1}\left(\mathscr{T} x_{0}\right)$ and $x_{2} \in \mathscr{R}^{-1}\left(\mathscr{T} x_{1}\right)$, using weakly increasing property of $\mathscr{T}$ with respect to $\mathscr{R}$, we obtain

$$
\mathscr{R} x_{1}=\mathscr{T} x_{0} \preceq \mathscr{T} x_{1}=\mathscr{R} x_{2} \preceq \mathscr{T} x_{2}=\mathscr{R} x_{3} .
$$


Continuing this process indefinitely, we get

$$
\mathscr{R} x_{1} \preceq \mathscr{R} x_{2} \preceq \mathscr{R} x_{3} \preceq \cdots \preceq \mathscr{R} x_{n} \preceq \mathscr{R} x_{n+1} \preceq \cdots .
$$

Now, we proceed to show that for $n \in \mathbb{N}$,

$$
d\left(\mathscr{R}\left(x_{n+1}\right), \mathscr{R}\left(x_{n+2}\right)\right) \leq\left(\frac{\beta}{1-\alpha}\right)^{n} d\left(\mathscr{R}\left(x_{1}\right), \mathscr{R}\left(x_{2}\right)\right) \text {. }
$$

As $\mathscr{R}\left(x_{2}\right) \geq \mathscr{R}\left(x_{1}\right)$, using (9), we have

$$
\begin{aligned}
d\left(\mathscr{R}\left(x_{2}\right), \mathscr{R}\left(x_{3}\right)\right) & \\
= & d\left(\mathscr{T}\left(x_{1}\right), \mathscr{T}\left(x_{2}\right)\right) \\
\leq & \alpha \frac{d\left(\mathscr{R}\left(x_{1}\right), \mathscr{T}\left(x_{1}\right)\right) \cdot d\left(\mathscr{R}\left(x_{2}\right), \mathscr{T}\left(x_{2}\right)\right)}{1+d\left(\mathscr{R}\left(x_{1}\right), \mathscr{R}\left(x_{2}\right)\right)} \\
+ & \beta d\left(\mathscr{R}\left(x_{1}\right), \mathscr{R}\left(x_{2}\right)\right), \\
& \quad\left(\text { owing to } d\left(\mathscr{R}\left(x_{1}\right), \mathscr{R}\left(x_{2}\right)\right)\right. \\
& \left.\leq 1+d\left(\mathscr{R}\left(x_{1}\right), \mathscr{R}\left(x_{2}\right)\right)\right) \\
\leq & \alpha d\left(\mathscr{R}\left(x_{2}\right), \mathscr{R}\left(x_{3}\right)\right)+\beta d\left(\mathscr{R}\left(x_{1}\right), \mathscr{R}\left(x_{2}\right)\right)
\end{aligned}
$$

which implies that

$$
(1-\alpha) d\left(\mathscr{R}\left(x_{2}\right), \mathscr{R}\left(x_{3}\right)\right) \leq \beta d\left(\mathscr{R}\left(x_{1}\right), \mathscr{R}\left(x_{2}\right)\right)
$$

so that

$$
d\left(\mathscr{R}\left(x_{2}\right), \mathscr{R}\left(x_{3}\right)\right) \leq\left(\frac{\beta}{1-\alpha}\right) d\left(\mathscr{R}\left(x_{1}\right), \mathscr{R}\left(x_{2}\right)\right) .
$$

Let (13) holds for all $n>0$. As $\mathscr{R}\left(x_{n+1}\right) \geq \mathscr{R}\left(x_{n}\right)$, using (9), we have

$$
\begin{aligned}
d\left(\mathscr{R}\left(x_{n+2}\right), \mathscr{R}\left(x_{n+3}\right)\right)=d\left(\mathscr{T}\left(x_{n+1}\right), \mathscr{T}\left(x_{n+2}\right)\right) \\
\leq \alpha \\
\left.+\beta d\left(\mathscr{R}\left(x_{n+1}\right), \mathscr{R}\left(x_{n+2}\right)\right), \mathscr{T}\left(x_{n+1}\right)\right) d\left(\mathscr{R}\left(x_{n+2}\right), \mathscr{T}\left(x_{n+2}\right)\right) \\
1+d\left(\mathscr{R}\left(x_{n+1}\right), \mathscr{R}\left(x_{n+2}\right)\right) \\
\left.\quad \text { owing to } d\left(\mathscr{R}\left(x_{n+1}\right), \mathscr{R}\left(x_{n+2}\right)\right)\right) \\
\leq 1+d\left(\mathscr{R}\left(x_{n+1}\right), \mathscr{R}\left(x_{n+2}\right)\right) \\
\leq \alpha d\left(\mathscr{R}\left(x_{n+2}\right), \mathscr{R}\left(x_{n+3}\right)\right) \\
+\beta d\left(\mathscr{R}\left(x_{n+1}\right), \mathscr{R}\left(x_{n+2}\right)\right),
\end{aligned}
$$

so that

$$
\begin{aligned}
d(\mathscr{R} & \left.\left(x_{n+2}\right), \mathscr{R}\left(x_{n+3}\right)\right) \\
& \leq\left(\frac{\beta}{1-\alpha}\right) d\left(\mathscr{R}\left(x_{n+1}\right), \mathscr{R}\left(x_{n+2}\right)\right) \\
& \leq\left(\frac{\beta}{1-\alpha}\right)^{n+1} d\left(\mathscr{R} x_{1}, \mathscr{R} x_{2}\right) .
\end{aligned}
$$

Now with $h=\beta /(1-\alpha)$, for any $m>n$, we have

$$
\begin{aligned}
& d\left(\mathscr{R}\left(x_{n}\right), \mathscr{R}\left(x_{m}\right)\right) \\
& \leq d\left(\mathscr{R}\left(x_{n}\right), \mathscr{R}\left(x_{n+1}\right)\right) \\
&+d\left(\mathscr{R}\left(x_{n+1}\right), \mathscr{R}\left(x_{n+2}\right)\right) \\
&+\cdots d\left(\mathscr{R}\left(x_{m-1}\right), \mathscr{R}\left(x_{m}\right)\right) \\
& \leq {\left[h^{n}+h^{n+1}+\cdots h^{m-1}\right] d\left(\mathscr{R} x_{1}, \mathscr{R} x_{2}\right) } \\
& \leq {\left[\frac{h^{n}}{1-h}\right] d\left(\mathscr{R} x_{1}, \mathscr{R} x_{2}\right) . }
\end{aligned}
$$

As $0<h=\beta /(1-\alpha)<1$, we have $\lim _{n \rightarrow+\infty} d\left(\mathscr{R}\left(x_{n}\right), \mathscr{R}\left(x_{m}\right)\right)=$ 0 , that is, $\left\{\mathscr{R} x_{n}\right\}$ is Cauchy sequences in the complete metric space $(\mathscr{X}, d)$ and hence there exists some $x \in \mathscr{X}$ such that

$$
\lim _{n \rightarrow+\infty} \mathscr{T}\left(x_{n}\right)=\lim _{n \rightarrow+\infty} \mathscr{R}\left(x_{n}\right)=x .
$$

Owing to commutativity of $\mathscr{T}$ with $\mathscr{R}$ and by using (10) (for each $n \geq 1$ ), we have

$$
\begin{aligned}
& \mathscr{R}\left(\mathscr{R} x_{n+1}\right)=\mathscr{R}\left(\mathscr{T} x_{n}\right)=\mathscr{T}\left(\mathscr{R} x_{n}\right), \\
& \text { that is } \mathscr{R}^{-1}\left(\mathscr{T}\left(\mathscr{R} x_{n}\right)\right)=\mathscr{R} x_{n+1} .
\end{aligned}
$$

Since $\mathscr{T}$ is weakly increasing with $\mathscr{R}$, we can write

$$
\mathscr{R}\left(\mathscr{R} x_{n+1}\right)=\mathscr{T}\left(\mathscr{R} x_{n}\right) \preceq \mathscr{T}\left(\mathscr{R} x_{n+1}\right)=\mathscr{R}\left(\mathscr{R} x_{n+2}\right)
$$

so that $\mathscr{R}\left(\mathscr{R} x_{n}\right)$ is nondecreasing. As the maps $\mathscr{R}$ and $\mathscr{T}$ are weakly reciprocally continuous, $\lim _{n \rightarrow \infty} \mathscr{R}\left(\mathscr{R} x_{n}\right)=$ $\lim _{n \rightarrow \infty} \mathscr{R}\left(\mathscr{T} x_{n-1}\right)=\mathscr{R} x$, which together with regularity of $\mathscr{X}$ gives rise to $\mathscr{R}\left(\mathscr{R} x_{n}\right) \preceq \mathscr{R} x$; that is, $\mathscr{R}\left(\mathscr{R} x_{n}\right)$ and $\mathscr{R} x$ are comparable. On using condition (9), we have

$$
\begin{aligned}
& d(\mathscr{R} x, \mathscr{T} x) \\
& \leq d\left(\mathscr{R} x, \mathscr{R}\left(\mathscr{R} x_{n+1}\right)\right)+d\left(\mathscr{R}\left(\mathscr{R} x_{n+1}\right), \mathscr{T} x\right) \\
&= d\left(\mathscr{R} x, \mathscr{R}\left(\mathscr{R} x_{n+1}\right)\right)+d\left(\mathscr{R}\left(\mathscr{T} x_{n}\right), \mathscr{T} x\right) \\
&= d\left(\mathscr{R} x, \mathscr{R}\left(\mathscr{R} x_{n+1}\right)\right)+d\left(\mathscr{T}\left(\mathscr{R} x_{n}\right), \mathscr{T} x\right) \\
&= d\left(\mathscr{R} x, \mathscr{R}\left(\mathscr{R} x_{n+1}\right)\right)+d\left(\mathscr{T} x, \mathscr{T}\left(\mathscr{R} x_{n}\right)\right) \\
& \leq d\left(\mathscr{R} x, \mathscr{R}\left(\mathscr{R} x_{n+1}\right)\right) \\
&+\alpha d(\mathscr{R} x, \mathscr{T} x) \frac{d\left(\mathscr{R}\left(\mathscr{R} x_{n}\right), \mathscr{T}\left(\mathscr{R} x_{n}\right)\right)}{1+d\left(\mathscr{R} x, \mathscr{R}\left(\mathscr{R} x_{n}\right)\right)} \\
&+\beta d\left(\mathscr{R} x, \mathscr{R}\left(\mathscr{R} x_{n}\right)\right) .
\end{aligned}
$$

On making $n \rightarrow \infty$ in the preceeding inequality, one gets

$$
d(\mathscr{R} x, \mathscr{T} x)=0,
$$

so that $\mathscr{T} x=\mathscr{R} x$. Thus, we have shown that $\mathscr{T}$ and $\mathscr{R}$ have a coincidence point. This completes the proof.

Setting $\alpha=0$ in Theorem 10, we deduce the following. 
Corollary 11. Let $(\mathscr{X}, \preceq)$ be a partially ordered set on which there is a metric $d$ on $\mathscr{X}$ such that $(\mathscr{X}, d)$ is a complete metric space. Let $\mathscr{T}, \mathscr{R}: \mathscr{X} \rightarrow \mathscr{X}$ be given mappings satisfying (for pairs $(x, y) \in \mathscr{X} \times \mathscr{X}$ wherein $\mathscr{R} x$ and $\mathscr{R} y$ are comparable)

$$
d(\mathscr{T} x, \mathscr{T} y) \leq \beta d(\mathscr{R} x, \mathscr{R} y)
$$

where $\beta$ is a nonnegative real number with $\beta<1$. Suppose that

(a) $\mathscr{X}$ is regular and $\mathscr{T}$ is weakly increasing with $\mathscr{R}$,

(b) the pair $(\mathscr{R}, \mathscr{T})$ is commuting as well as weakly reciprocally continuous.

Then $\mathscr{T}$ and $\mathscr{R}$ have a coincidence point. That is, there exists $u \in \mathscr{X}$ such that $\mathscr{R} u=\mathscr{T} u$.

Theorem 12. Let $(\mathscr{X}, \preceq)$ be a partially ordered set equipped with a metric $d$ on $\mathscr{X}$ such that $(\mathscr{X}, d)$ is a complete metric space. Let $\mathscr{T}, \mathscr{R}: \mathscr{X} \rightarrow \mathscr{X}$ be two mappings satisfying (for pairs $(x, y) \in \mathscr{X} \times \mathscr{X}$ wherein $\mathscr{R} x$ and $\mathscr{R} y$ are comparable)

$$
\begin{aligned}
d(\mathscr{T} x, \mathscr{T} y) \leq & \alpha \frac{d(\mathscr{R} x, \mathscr{T} x) \cdot d(\mathscr{R} y, \mathscr{T} y)}{1+d(\mathscr{R} x, \mathscr{R} y)} \\
& +\beta d(\mathscr{R} x, \mathscr{R} y),
\end{aligned}
$$

where $\alpha, \beta$ are non-negative real numbers with $\alpha+\beta<1$. Suppose that

(a) $\mathscr{T}$ is weakly increasing with $\mathscr{R}$,

(b) the pair $\{\mathscr{T}, \mathscr{R}\}$ is compatible and reciprocally continuous.

Then $\mathscr{T}$ and $\mathscr{R}$ have a coincidence point. That is, there exists $u \in \mathscr{X}$ such that $\mathscr{R} u=\mathscr{T} u$.

Proof. Proceeding on the lines of the proof of Theorem 10, one can furnish a sequence $\left\{x_{n}\right\}$ such that

$$
\lim _{n \rightarrow+\infty} \mathscr{T}\left(x_{n}\right)=\lim _{n \rightarrow+\infty} \mathscr{R}\left(x_{n}\right)=x
$$

Now, it remains to show that $x$ is a coincidence point of $\mathscr{T}$ and $\mathscr{R}$. To accomplish this, as the pair $\{\mathscr{T}, \mathscr{R}\}$ is compatible as well as reciprocally continuous,

$$
\begin{gathered}
\lim _{n \rightarrow \infty} d\left(\mathscr{R}\left(\mathscr{T}\left(x_{n}\right)\right), \mathscr{T}\left(\mathscr{R}\left(x_{n}\right)\right)\right)=0, \\
\mathscr{R}(x)=\lim _{n \rightarrow \infty} \mathscr{R}\left(\mathscr{T}\left(x_{n}\right)\right), \quad \mathscr{T}(x)=\lim _{n \rightarrow \infty} \mathscr{T}\left(\mathscr{R}\left(x_{n}\right)\right),
\end{gathered}
$$

whenever

$$
\lim _{n \rightarrow+\infty} \mathscr{T}\left(x_{n}\right)=\lim _{n \rightarrow+\infty} \mathscr{R}\left(x_{n}\right)=x
$$

By using (29) in (28), we have $d(\mathscr{T} x, \mathscr{R} x)=0$, so that $\mathscr{T} x=$ $\mathscr{R} x$.

By appealing Theorems 10 and 12, one can also have the following natural theorem.
Theorem 13. Let $(\mathscr{X}, \preceq)$ be a partially ordered set on which there is a metric $d$ on $\mathscr{X}$ such that $(\mathscr{X}, d)$ is a complete metric space. Let $\mathscr{T}: \mathscr{X} \rightarrow \mathscr{X}$ be nondecreasing mapping satisfying (for pairs $(x, y) \in \mathscr{X} \times \mathscr{X}$ wherein $\mathscr{R} x$ and $\mathscr{R} y$ are comparable)

$$
d(\mathscr{T} x, \mathscr{T} y) \leq \alpha \frac{d(x, \mathscr{T} x) \cdot d(y, \mathscr{T} y)}{1+d(x, y)}+\beta d(x, y),
$$

where $\alpha, \beta$ are non-negative real numbers such that $\alpha+\beta<1$. Suppose that

(I) $\mathscr{T} x \preceq \mathscr{T}(\mathscr{T} x)$ for all $x \in \mathscr{X}$,

(II) either $\mathscr{T}$ is continuous or $\mathscr{X}$ is regular.

Then, $\mathscr{T}$ has a fixed point.

Proof. The proof of this theorem can be outlined on the lines of the proof of Theorem 10 realizing $\mathscr{R}$ to be the identity mapping on $\mathscr{X}$.

Now, we introduce the following property which will be utilized in our next theorem.

Property $(A)$. If $\mathscr{R}\left(x_{n}\right)$ is a nondecreasing sequence in $X$ such that $\lim _{n \rightarrow+\infty} \mathscr{R}\left(x_{n}\right)=x$, then $\mathscr{R}\left(x_{n}\right)$ is comparable to $\mathscr{R}(x)$, for all $n \in N$.

Theorem 14. Let $(\mathscr{X}, \preceq)$ be a partially ordered set on which there exists a metric $d$ on $\mathscr{X}$ such that $(\mathscr{X}, d)$ is a complete metric space. Let $\mathscr{T}, \mathscr{R}: \mathscr{X} \rightarrow \mathscr{X}$ be given mappings satisfying (for pairs $(x, y) \in \mathscr{X} \times \mathscr{X}$ wherein $\mathscr{R} x$ and $\mathscr{R} y$ are comparable)

$$
\begin{aligned}
d(\mathscr{T} x, \mathscr{T} y) \leq & \alpha \frac{d(\mathscr{R} x, \mathscr{T} x) \cdot d(\mathscr{R} y, \mathscr{T} y)}{1+d(\mathscr{R} x, \mathscr{R} y)} \\
& +\beta d(\mathscr{R} x, \mathscr{R} y),
\end{aligned}
$$

where $\alpha, \beta$ are non-negative real numbers with $\alpha+\beta<1$. Suppose that

(a) $\mathscr{X}$ is regular and $\mathscr{T}$ is weakly increasing with $\mathscr{R}$,

(b) the pair $(\mathscr{R}, \mathscr{T})$ is commuting as well as weakly reciprocally continuous,

(c) $\mathscr{R}$ satisfies Property (A).

Then $\mathscr{R}$ and $\mathscr{T}$ have a common fixed point.

Proof. Proceeding on the lines of the proof of Theorem 10, one can inductively construct nondecreasing sequence $\left\{\mathscr{R} x_{n}\right\}$, such that $\lim _{n \rightarrow \infty} \mathscr{T} x_{n}=\lim _{n \rightarrow \infty} \mathscr{R} x_{n+1}=x$, and $\mathscr{R}(x)=\mathscr{T}(x)$. Since $\mathscr{R}\left(x_{n}\right)$ and $\mathscr{R}(x)$ are comparable (for all $n \in N)$, by using condition (32) we have

$$
\begin{aligned}
d\left(\mathscr{R} x, \mathscr{R} x_{n+1}\right)= & d\left(\mathscr{T} x, \mathscr{T} x_{n}\right) \\
\leq & \alpha d(\mathscr{R} x, \mathscr{T} x) \frac{d\left(\mathscr{R} x_{n}, \mathscr{T} x_{n}\right)}{1+d\left(\mathscr{R} x, \mathscr{R} x_{n}\right)} \\
& +\beta d\left(\mathscr{R} x, \mathscr{R} x_{n}\right) .
\end{aligned}
$$


Taking the limit as $n \rightarrow \infty$, one gets

$$
x=\mathscr{R} x=\mathscr{T} x .
$$

This completes the proof.

Theorem 15. Let $(\mathscr{X}, \preceq)$ be a partially ordered set on which there exists a metric $d$ on $\mathscr{X}$ such that $(\mathscr{X}, d)$ is a complete metric space. Let $\mathscr{T}, \mathscr{R}: \mathscr{X} \rightarrow \mathscr{X}$ be given mappings satisfying (for pairs $(x, y) \in \mathscr{X} \times \mathscr{X}$ wherein $\mathscr{R} x$ and $\mathscr{R} y$ are comparable)

$$
\begin{aligned}
d(\mathscr{T} x, \mathscr{T} y) \leq & \alpha \frac{d(\mathscr{R} x, \mathscr{T} x) \cdot d(\mathscr{R} y, \mathscr{T} y)}{1+d(\mathscr{R} x, \mathscr{R} y)} \\
& +\beta d(\mathscr{R} x, \mathscr{R} y),
\end{aligned}
$$

where $\alpha, \beta$ are non-negative real numbers with $\alpha+\beta<1$. Suppose that

(a) $\mathscr{T}$ is weakly increasing with $\mathscr{R}$,

(b) the pair $\{\mathscr{T}, \mathscr{R}\}$ is compatible and reciprocally continuous,

(c) $\mathscr{R}$ satisfies Property (A).

Then $\mathscr{R}$ and $\mathscr{T}$ have a common fixed point.

Proof. Proof is obvious in view of Theorems 12 and 14.

\section{Uniqueness Results}

In what follows, we investigate the conditions under which Theorem 10 ensures the uniqueness of common fixed point.

Theorem 16. If, in addition to the hypotheses of Theorem 10, every $\left(x, x^{*}\right) \in \mathscr{X} \times \mathscr{X}$, there exists a $u \in \mathscr{X}$ such that $\mathscr{T} u$ is upper bound of $\mathscr{T} x$ and $\mathscr{T} x^{*}$, then $\mathscr{T}$ and $\mathscr{R}$ have a unique common fixed point.

Proof. In view of Theorem 10, the set of coincidence points of the maps $\mathscr{T}$ and $\mathscr{R}$ is non-empty. If $x$ and $x^{*}$ are two coincidence points of the maps $\mathscr{T}$ and $\mathscr{R}$ (i.e., $\mathscr{R} x=$ $\left.\mathscr{T} x, \mathscr{R} x^{*}=\mathscr{T} x^{*}\right)$, then we proceed to show that

$$
\mathscr{R} x=\mathscr{R} x^{*} .
$$

In view of the additional hypothesis of this theorem, there exists $u \in \mathscr{X}$ such that $\mathscr{T} u$ is upper bound of $\mathscr{T} x$ and $\mathscr{T} x^{*}$. Put $u_{0}=u$, and choose $u_{1} \in \mathscr{X}$ such that $\mathscr{R} u_{1}=\mathscr{T} u_{0}$. Now, proceeding on the lines of the proof of Theorem 10, one can inductively define sequence $\left\{\mathscr{R} u_{n}\right\}$ such that

$$
\mathscr{R} u_{n}=\mathscr{T} u_{n-1} \quad \forall n,
$$

wherein

$$
\mathscr{R} u_{1} \preceq \mathscr{R} u_{2} \preceq \mathscr{R} x_{3} \preceq \cdots \preceq \mathscr{R} u_{n} \preceq \mathscr{R} u_{n+1} \preceq \cdots .
$$

Further, setting $x_{0}=x$, and $x_{0}^{*}=x^{*}$, one can also define the sequences $\left\{\mathscr{R} x_{n}\right\}$ and $\left\{\mathscr{R} x_{n}^{*}\right\}$ such that

$$
\mathscr{R} x_{n}=\mathscr{T} x_{n-1}, \quad \mathscr{R} x_{n}^{*}=\mathscr{T} x_{n-1}^{*} .
$$

For every $n \geq 1$, we have

$$
\mathscr{R} x_{n}=\mathscr{T} x, \quad \mathscr{R} x_{n}^{*}=\mathscr{T} x^{*} \quad \forall n \geq 1 .
$$

Since $\mathscr{T} u=\mathscr{R} u_{1}$ is upper bound of $\mathscr{T} x=\mathscr{R} x_{1}$ and $\mathscr{T} x^{*}=$ $\mathscr{R} x_{1}^{*}$, then

$$
\mathscr{R} x_{1}=\mathscr{R} x \preceq \mathscr{R} u_{1}, \quad \mathscr{R} x_{1}^{*}=\mathscr{R} x^{*} \preceq \mathscr{R} u_{1} .
$$

It is easy to show that $\mathscr{R} x \preceq \mathscr{R} u_{n}$ and $\mathscr{R} x^{*} \preceq \mathscr{R} u_{n}$ for all $n \geq 1$, then $\mathscr{R} x$ and $\mathscr{R} x^{*}$ are comparable with $\mathscr{R} u_{n}$. On using (9), we have

$$
\begin{aligned}
d\left(\mathscr{R} x, \mathscr{R} u_{n+1}\right)= & d\left(\mathscr{T} x, \mathscr{T} u_{n}\right) \\
\leq & \alpha d(\mathscr{R} x, \mathscr{T} x) \frac{d\left(\mathscr{R} u_{n}, \mathscr{T} u_{n}\right)}{1+d\left(\mathscr{R} x, \mathscr{R} u_{n}\right)} \\
& +\beta d\left(\mathscr{R} x, \mathscr{R} u_{n}\right)
\end{aligned}
$$

or

$$
d\left(\mathscr{R} x, \mathscr{R} u_{n+1}\right) \leq \beta d\left(\mathscr{R} x, \mathscr{R} u_{n}\right) .
$$

Owing to (43), we can write

$$
\begin{gathered}
d\left(\mathscr{R} x, \mathscr{R} u_{n+1}\right) \leq \beta d\left(\mathscr{R} x, \mathscr{R} u_{n}\right) \\
\leq \beta^{2} d\left(\mathscr{R} x, \mathscr{R} u_{n-1}\right) \leq \cdots \\
\leq \beta^{n+1} d\left(\mathscr{R} x, \mathscr{R} u_{0}\right) .
\end{gathered}
$$

Taking the limit as $n \rightarrow \infty$ in (44), we get

$$
\lim _{n \rightarrow \infty} d\left(\mathscr{R} x, \mathscr{R} u_{n}\right)=0 \text {, }
$$

as $0<\beta<1$.

Similarly, one can also show that

$$
\lim _{n \rightarrow \infty} d\left(\mathscr{R} x^{*}, \mathscr{R} u_{n}\right)=0 .
$$

On using (45) and (46), we can have

$$
d\left(\mathscr{R} x, \mathscr{R} x^{*}\right) \leq d\left(\mathscr{R} x, \mathscr{R} u_{n+1}\right)+d\left(\mathscr{R} u_{n+1}, \mathscr{R} x^{*}\right)
$$

so that $\lim _{n \rightarrow \infty} d\left(\mathscr{R} x, \mathscr{R} x^{*}\right) 0$, that is, $\mathscr{R} x=\mathscr{R} x^{*}$. Thus, we have proved (36).

Since $\mathscr{R} x=\mathscr{T} x$, owing to commutativity of $\mathscr{T}$ and $\mathscr{R}$, one can write

$$
\mathscr{R}(\mathscr{R} x)=\mathscr{R}(\mathscr{T} x)=\mathscr{T}(\mathscr{R} x)
$$

which on inserting $\mathscr{R} x=z$ gives rise to

$$
\mathscr{R} z=\mathscr{T} z \text {. }
$$

Thus, $z$ is another coincidence point of the pair. Now, due to $\mathscr{R} x=\mathscr{R} x^{*}$ (for every coincidence point $x$ and $x^{*}$ ) and owing to the fact that $z$ is coincidence point of the pair $\{\mathscr{R}, \mathscr{T}\}$, it follows that $\mathscr{R} z=\mathscr{R} x$, that is,

$$
\mathscr{R} z=z .
$$


On making use of (49) and (50), we can have

$$
z=\mathscr{R} z=\mathscr{T} z
$$

which shows that $z$ is a common fixed point of $\mathscr{T}$ and $\mathscr{R}$.

To prove the uniqueness, let $p$ be another common fixed point of the pair $\{\mathscr{R}, \mathscr{T}\}$. Since $p$ and $z$ are coincidence points, then $\mathscr{R} p=\mathscr{R} z$.

Owing to that $p$ and $z$ are common fixed point of the pairs $\{\mathscr{R}, \mathscr{T}\}$, one can have

$$
p=\mathscr{R} p=\mathscr{R} z=z .
$$

This completes the proof.

The following simple example demonstrates Theorem 16.

Example 17. Consider $\mathscr{X}=\{(1,0),(0,1)\} \subset R^{2}$ equipped with natural order $(x, y) \leq(z, t) \Leftrightarrow x \leq z$ and $y \leq t$. Thus $(\mathscr{X}, \leq)$, is a partially ordered set wherein the two elements are not comparable to each other. Also, $(\mathscr{X}, \leq)$ is a complete metric space under Euclidean metric. Defined mappings $\mathscr{T}$ and $\mathscr{R}$ : $\mathscr{X} \rightarrow \mathscr{X}$ as

$$
\begin{aligned}
& \mathscr{T}(x, y)= \begin{cases}(1,0), & (x, y)=(1,0) \\
(1,0), & (x, y)=(0,1),\end{cases} \\
& \mathscr{R}(x, y)= \begin{cases}(1,0), & (x, y)=(1,0) \\
(0,1), & (x, y)=(0,1) .\end{cases}
\end{aligned}
$$

As $\mathscr{R}(x, y)$ and $\mathscr{R}(z, t)$ in $\mathscr{X}$ are merely comparable to themselves, inequality (9) is vacuously satisfied for every $\alpha, \beta$.

Notice that $\mathscr{T}(\mathscr{X})=(1,0) \subset \mathscr{R}(\mathscr{X})=\mathscr{X}$. Also, for $(x, y)=(1,0)$,

$$
\mathscr{R}(z, t)=\mathscr{T}(x, y)=\mathscr{T}(1,0)=(1,0) \Longrightarrow(z, t)=(1,0)
$$

so that

$$
\mathscr{T}(1,0)=\mathscr{T}(x, y) \leq \mathscr{T}(z, t)=\mathscr{T}(1,0) .
$$

Otherwise, for $(z, t)=(0,1)$,

$$
\mathscr{R}(z, t)=\mathscr{T}(x, y)=\mathscr{T}(0,1)=(1,0) \Longrightarrow(z, t)=(1,0)
$$

so that

$$
(1,0)=\mathscr{T}(0,1)=\mathscr{T}(x, y) \leq \mathscr{T}(z, t)=\mathscr{T}(1,0)=(1,0) .
$$

Thus $\mathscr{T}$ is weakly increasing with respect to $\mathscr{R}$.

If $\left\{\left(x_{n}, y_{n}\right)\right\} \subset \mathscr{X}$ is a nondecreasing sequence converging to $(x, y) \in \mathscr{X}$, then necessarily $\left\{\left(x_{n}, y_{n}\right)\right\}$ must be constant sequence, that is, $\left(x_{n}, y_{n}\right)=(x, y)$, for all $n \in N$, so that limit $(x, y)$ is an upper bond for all the terms in the sequence which shows that $\mathscr{X}$ is regular.

By using definitions of the maps $\mathscr{T}$ and $\mathscr{R}$, we have

$$
(x, y)=\mathscr{R}(x, y)=\lim _{n \rightarrow \infty} \mathscr{T}\left(x_{n}, y_{n}\right)=\lim _{n \rightarrow \infty} \mathscr{R} \mathscr{T}\left(x_{n}, y_{n}\right)
$$

wherein $\lim _{n \rightarrow \infty} \mathscr{R}\left(x_{n}, y_{n}\right)=\lim _{n \rightarrow \infty} \mathscr{T}\left(x_{n}, y_{n}\right)=(x, y)$ so that

$$
\mathscr{R}(x, y)=\lim _{n \rightarrow \infty} \mathscr{R} \mathscr{T}\left(x_{n}, y_{n}\right) \text {, }
$$

which shows that the pair $\{\mathscr{R}, \mathscr{T}\}$ is weakly reciprocally continuous. Also, the pair $\{\mathscr{R}, \mathscr{T}\}$ is clearly commuting.

Now, we show that for every $x$ and $x^{*}$ in $\mathscr{X}$, there exists a $u \in \mathscr{X}$ such that $\mathscr{T} u$ is upper bound of $\mathscr{T} x$ and $\mathscr{T} x^{*}$. If $x=x^{*}$, then choice of $u$ is obvious. Otherwise, if $x=(1,0)$ and $x^{*}=(0,1)$, we can choose $u=(0,1)$ such that $\mathscr{T} u=$ $\mathscr{T}(0,1)=(1,0)$ is upper bound of $\mathscr{T} x=(1,0)$ and $\mathscr{T} x^{*}=$ $(1,0)$.

Thus, we have shown that all the conditions of Theorem 16 are satisfied and $(x, y)=(1,0)$ is the unique common fixed point of $\mathscr{T}$ and $\mathscr{R}$.

Theorem 18. If, in addition to the hypotheses of Theorem 12, for pairs $\left(x, x^{*}\right) \in \mathscr{X} \times \mathscr{X}$, there exists a $u \in \mathscr{X}$ such that $\mathscr{T} u$ is upper bound of $\mathscr{T} x$ and $\mathscr{T} x^{*}$, then $\mathscr{T}$ and $\mathscr{R}$ have a unique common fixed point.

Proof. Proof is obvious in view of Theorems 12 and 16.

Corollary 19. In addition to the hypotheses of Theorem 16 (or Theorem 18), suppose that for every $\left(x, x^{*}\right) \in \mathscr{X} \times \mathscr{X}$ there exists a $u \in \mathscr{X}$ such that $\mathscr{T} u$ is upper bound of $\mathscr{T} x$ and $\mathscr{T} x^{*}$. Then $\mathscr{T}$ has a unique fixed point; that is, there exists a unique $x \in \mathscr{X}$ such that $x=\mathscr{T} x$.

Proof. In Theorem 16, if $\mathscr{R}=I$ (the identity mapping on $X$ ), we have the result.

The following example demonstrates Theorem 18.

Example 20. Consider $\mathscr{X}=[0, \infty)$ equipped with the usual metric and natural order $\leq$. Define two mappings $\mathscr{T}, \mathscr{R}$ : $x \rightarrow \mathscr{X}$ by

$$
\begin{gathered}
\mathscr{T} x= \begin{cases}x^{3}+2, & 0 \leq x<1 \\
3, & 1 \leq x,\end{cases} \\
\mathscr{R} x= \begin{cases}2 x^{3}+1, & 0 \leq x<1 \\
3, & 1 \leq x .\end{cases}
\end{gathered}
$$

Then, evidently $(\mathscr{X}, \leq)$ is a partially ordered set and the maps $\mathscr{T}$ and $\mathscr{R}$ satisfy inequality (9) with $\beta=1 / 2$ and $\alpha=1 / 3$, for $x, y \in[0, \infty)$.

To show that $\mathscr{T}$ is weakly increasing with respect to $\mathscr{R}$, notice that $\mathscr{T}(\mathscr{X})=[2,3] \subset[1,3]=\mathscr{R}(\mathscr{X})$. Firstly, we argue the case $x=0$. Let $y \in \mathscr{R}^{-1}(T 0)$, that is, $\mathscr{R}(y)=\mathscr{T}(0)=2$ so that $2 y^{3}+1=2$ or $y=1 / 2^{1 / 3}$. Using definitions of $\mathscr{T}$ and $\mathscr{R}$, we have

$$
2=\mathscr{T}(0) \leq \mathscr{T}(y)=\mathscr{T}\left(\frac{1}{2^{1 / 3}}\right)=\frac{1}{2}+2 .
$$

Secondly, if $x \geq 1$, then we have $\mathscr{R}(y)=\mathscr{T}(x)=3$. By using definition of $\mathscr{R}$, we have $y \geq 1$, so that

$$
3=\mathscr{T}(x) \leq \mathscr{T}(y)=3 .
$$


Finally, we consider the case $0<x<1$. Let $y \in$ $\mathscr{R}^{-1}(\mathscr{T}(x))$, that is, $\mathscr{R}(y)=\mathscr{T}(x)$. In view of definition of $\mathscr{T}$, we have $\mathscr{T}(x)=x^{3}+2$, so that $\mathscr{R}(y)=x^{3}+2$. By using the definition of $\mathscr{R}$, we have $\mathscr{R}(y)=2 y^{3}+1$, so that $2 y^{3}+1=x^{3}+2$ or $y=\left(\left(x^{3}+1\right) / 2\right)^{1 / 3}$. Thus, we have

$$
x^{3}+2=\mathscr{T}(x) \leq \mathscr{T}(y)=\mathscr{T}\left(\left(\frac{x^{3}+1}{2}\right)^{1 / 3}\right)=\frac{x^{3}+1}{2}+2 .
$$

Therefore $\mathscr{T}$ is weakly increasing with respect to $\mathscr{R}$.

Since $\mathscr{T}$ and $\mathscr{R}$, are continuous, therefore this pair of maps is reciprocally continuous.

Now, we show that maps $\mathscr{T}$ and $\mathscr{R}$, are compatible. If $\lim _{n \rightarrow \infty} x_{n}=k$, and $k \geq 1$, then $\lim _{n \rightarrow \infty} \mathscr{T} x_{n}=$ $\lim _{n \rightarrow \infty} \mathscr{R} x_{n}=3$ and henceforth

$$
\lim _{n \rightarrow \infty} \mathscr{T} \mathscr{R} x_{n}=\lim _{n \rightarrow \infty} \mathscr{R} \mathscr{T} x_{n}=3
$$

implying thereby $\lim _{n \rightarrow \infty} d\left(\mathscr{R} \mathscr{T} x_{n}, \mathscr{T} \mathscr{R} x_{n}\right)=0$ so that $\mathscr{T}$ and $\mathscr{R}$, are compatible.

Thus, we have shown that all the conditions of Theorem 18 are satisfied and $x=3$ is the unique fixed point of $\mathscr{T}$ and $\mathscr{R}$.

\section{An Application}

In this section, we present an application of Theorem 13 and used the idea of Ćirić et al. [29] to define a partial order and prove an existence theorem for the solution of an integral equation.

Theorem 21. Consider the integral equation

$$
x(t)=\int_{0}^{T} K(t, s, x(s)) d s+g(t), \quad t \in[0, T]
$$

with $T>0$ wherein

(i) the functions $K:[0, T] \times[0, T] \times \mathbb{R}^{n} \rightarrow \mathbb{R}^{n}$ and $g$ : $\mathbb{R}^{n} \rightarrow \mathbb{R}^{n}$ are continuous,

(ii) for each $t, s \in[0, T]$,

$$
K(t, s, x(s)) \ll K\left(t, s, \int_{0}^{T} K(s, \tau, x(\tau)) d \tau+g(s)\right),
$$

where $\ll$ denotes a partial order relation on $\mathbb{R}^{n}$,

(iii) there exists a continuous function $p:[0, T] \times[0, T] \rightarrow$ $\mathbb{R}_{+}$such that

$$
|K(t, s, u)-K(t, s, v)| \leq p(t, s)|u-v|
$$

for each $t, s \in[0, T]$ and is also comparable $u, v \in \mathbb{R}^{n}$,

(iv) $\sup _{t \in[0, T]} \int_{0}^{T} p(t, s) d s \leq \beta<1$.

Then the integral equation (66) has a unique solution $x^{*}$ in $C\left([0, T], \mathbb{R}^{n}\right)$.
Proof. Consider $\mathscr{X}:=C\left([0, T], \mathbb{R}^{n}\right)$ with the usual supremum norm, that is,

$$
\|x\|=\max _{t \in[0, T]}|x(t)|,
$$

for $x, y \in C\left([0, T] \mathbb{R}^{n}\right)$. Define on $\mathscr{X}$ a partial order as follows $\left(x, y \in C\left([0, T], \mathbb{R}^{n}\right)\right)$ :

$$
x \preceq y \quad \text { iff } x(t) \ll y(t) \text { for any } t \in[0, T] .
$$

Then $(\mathscr{X}, \preceq)$ is a partially ordered set and $(\mathscr{X},\|\cdot\|)$ is a complete metric space.

Moreover for any increasing sequence $\left\{x_{n}\right\}$ in $\mathscr{X}$ converging to $x^{*} \in \mathscr{X}$, we have $x_{n}(t) \ll x^{*}(t)$ for any $t \in[0, T]$. Also for every $x, y \in \mathscr{X}$ there exists $c \in \mathscr{X}$ which depends on $x$ and $y$ and is also comparable to $x$ and $y$ (cf. [21]).

Define $\mathscr{T}: C([0, T]) \rightarrow C([0, T])$ by

$$
\begin{array}{r}
\mathscr{T} u(t)=\int_{0}^{T} K(t, s, u(s)) d s+g(t), \\
t \in[0, T], u \in C([0, T]) .
\end{array}
$$

Let us prove that

$$
\mathscr{T} u \preceq \mathscr{T}(\mathscr{T} u) \quad \forall u \in C([0, T]) .
$$

Let $u \in C([0, T])$. From (ii), for all $t \in[0, T]$, we have

$\mathscr{T} u(t)$

$$
\begin{aligned}
& =\int_{0}^{T} K(t, s, u(s)) d s+g(t) \\
& \ll \int_{0}^{T} K\left(t, s, \int_{0}^{T} K(s, \tau, u(\tau)) d \tau+g(s)\right) d s+g(t) \\
& =\int_{0}^{T} K(t, s, \mathscr{T} u(s)) d s+g(t) \\
& =\mathscr{T}(\mathscr{T} u)(t) .
\end{aligned}
$$

Then (72) holds.

Now, for all $x, y \in C([0, T])$ with $y \preceq x$, we have (by (iii))

$$
\begin{aligned}
& |\mathscr{T} x(t)-\mathscr{T} y(t)| \\
& \leq \int_{0}^{T}|K(t, s, x(s))-K(t, s, y(s))| d s \\
& \quad \leq \int_{0}^{T} p(t, s)(|x(s)-y(s)|) d s \\
& \quad \leq\|x-y\| \int_{0}^{T} p(t, s) d s .
\end{aligned}
$$

Hence

$$
\begin{aligned}
& \|\mathscr{T} x(t)-\mathscr{T} y(t)\| \\
& \quad \leq\|x-y\| \sup _{t \in[0, T]} \int_{0}^{T} p(t, s) d s \\
& \quad \leq \beta\|x-y\| .
\end{aligned}
$$


On the other hand, it is demonstrated in [16] that condition of regularity is satisfied for $\mathscr{X}=C\left([0, T] ; \mathbb{R}^{n}\right)$.

Thus, all the hypotheses of Theorem 13 are satisfied for $\alpha=0$, and then $\mathscr{T}$ has a fixed point $u^{*} \in C\left([0, T] ; \mathbb{R}^{n}\right)$; that is, $u^{*}$ is a solution of the integral equation (66).

\section{Acknowledgments}

The authors would like to thank the referees for careful reading and for providing valuable suggestions and comments for this paper. Also, Poom Kumam would like to thank the King Mongkuts University of Technology Thonburi (KMUTT) for the financial support and Dhananjay Gopal is thankful to CSIR, Government of India, Grant no. 25(0215)/13/EMR-II.

\section{References}

[1] S. Banach, "Sur les opérations dans les ensembles abstraits et leur application aux équations intégrales," Fundamenta Mathematicae, vol. 3, pp. 133-181, 1922.

[2] H. K. Nashine and I. Altun, "Fixed point theorems for generalized weakly contractive condition in ordered metric spaces," Fixed Point Theory and Applications, vol. 2011, Article ID 132367, 20 pages, 2011.

[3] R. P. Agarwal, M. A. El-Gebeily, and D. O’Regan, "Generalized contractions in partially ordered metric spaces," Applicable Analysis, vol. 87, no. 1, pp. 109-116, 2008.

[4] I. Altun and H. Simsek, "Some fixed point theorems on ordered metric spaces and application," Fixed Point Theory and Applications, vol. 2010, Article ID 621469, 17 pages, 2010.

[5] A. Amini-Harandi and H. Emami, "A fixed point theorem for contraction type maps in partially ordered metric spaces and application to ordinary differential equations," Nonlinear Analysis. Theory, Methods \& Applications, vol. 72, no. 5, pp. 2238-2242, 2010.

[6] I. Beg and A. R. Butt, "Fixed point for set-valued mappings satisfying an implicit relation in partially ordered metric spaces," Nonlinear Analysis. Theory, Methods \& Applications, vol. 71, no. 9, pp. 3699-3704, 2009.

[7] T. G. Bhaskar and V. Lakshmikantham, "Fixed point theorems in partially ordered metric spaces and applications," Nonlinear Analysis. Theory, Methods \& Applications, vol. 65, no. 7, pp. 13791393, 2006.

[8] A. Cabada and J. J. Nieto, "Fixed points and approximate solutions for nonlinear operator equations," Journal of Computational and Applied Mathematics, vol. 113, no. 1-2, pp. 1725,2000 , Fixed point theory with applications in nonlinear analysis.

[9] J. Caballero, J. Harjani, and K. Sadarangani, "Contractive-like mapping principles in ordered metric spaces and application to ordinary differential equations," Fixed Point Theory and Applications, vol. 2010, Article ID 916064, 14 pages, 2010.

[10] L. Ćirić, N. Cakić, M. Rajović, and J. S. Ume, "Monotone generalized nonlinear contractions in partially ordered metric spaces," Fixed Point Theory and Applications, vol. 2008, Article ID 131294, 11 pages, 2008.

[11] Lj. B. Ćirić, D. Miheţ, and R. Saadati, "Monotone generalized contractions in partially ordered probabilistic metric spaces," Topology and its Applications, vol. 156, no. 17, pp. 2838-2844, 2009.
[12] J. Harjani and K. Sadarangani, "Fixed point theorems for weakly contractive mappings in partially ordered sets," Nonlinear Analysis. Theory, Methods \& Applications, vol. 71, no. 7-8, pp. 34033410, 2009.

[13] J. Harjani and K. Sadarangani, "Generalized contractions in partially ordered metric spaces and applications to ordinary differential equations," Nonlinear Analysis. Theory, Methods \& Applications, vol. 72, no. 3-4, pp. 1188-1197, 2010.

[14] H. K. Nashine and B. Samet, "Fixed point results for mappings satisfying $(\psi, \varphi)$-weakly contractive condition in partially ordered metric spaces," Nonlinear Analysis. Theory, Methods \& Applications, vol. 74, no. 6, pp. 2201-2209, 2011.

[15] H. K. Nashine and W. Shatanawi, "Coupled common fixed point theorems for a pair of commuting mappings in partially ordered complete metric spaces," Computers \& Mathematics with Applications, vol. 62, no. 4, pp. 1984-1993, 2011.

[16] J. J. Nieto and R. Rodríguez-López, "Contractive mapping theorems in partially ordered sets and applications to ordinary differential equations," Order, vol. 22, no. 3, pp. 223-239, 2005.

[17] J. J. Nieto and R. Rodríguez-López, "Existence and uniqueness of fixed point in partially ordered sets and applications to ordinary differential equations," Acta Mathematica Sinica, vol. 23, no. 12, pp. 2205-2212, 2007.

[18] R. P. Pant, "Common fixed points of four mappings," Bulletin of the Calcutta Mathematical Society, vol. 90, no. 4, pp. 281-286, 1998.

[19] R. P. Pant, R. K. Bisht, and D. Arora, "Weak reciprocal continuity and fixed point theorems," Annali dell'Universitá di Ferrara. Sezione VII. Scienze Matematiche, vol. 57, no. 1, pp. 181-190, 2011.

[20] A. C. M. Ran and M. C. B. Reurings, "A fixed point theorem in partially ordered sets and some applications to matrix equations," Proceedings of the American Mathematical Society, vol. 132, no. 5, pp. 1435-1443, 2004.

[21] D. O’Regan and A. Petruşel, "Fixed point theorems for generalized contractions in ordered metric spaces," Journal of Mathematical Analysis and Applications, vol. 341, no. 2, pp. 12411252, 2008.

[22] B. Samet, "Coupled fixed point theorems for a generalized Meir-Keeler contraction in partially ordered metric spaces," Nonlinear Analysis. Theory, Methods \& Applications, vol. 72, no. 12, pp. 4508-4517, 2010.

[23] W. Sintunavarat, Y. J. Cho, and P. Kumam, "Urysohn integral equations approach by common fixed points in complex valued metric spaces," Advances in Difference Equations, vol. 2013, article 49, 2013.

[24] W. Shatanawi, "Partially ordered cone metric spaces and coupled fixed point results," Computers \& Mathematics with Applications, vol. 60, no. 8, pp. 2508-2515, 2010.

[25] J. Harjani, B. López, and K. Sadarangani, "A fixed point theorem for mappings satisfying a contractive condition of rational type on a partially ordered metric space," Abstract and Applied Analysis, vol. 2010, Article ID 190701, 8 pages, 2010.

[26] D. S. Jaggi, "Some unique fixed point theorems," Indian Journal of Pure and Applied Mathematics, vol. 8, no. 2, pp. 223-230, 1977.

[27] B. C. Dhage, "Condensing mappings and applications to existence theorems for common solution of differential equations," Bulletin of the Korean Mathematical Society, vol. 36, no. 3, pp. 565-578, 1999.

[28] B. C. Dhage, D. O'Regan, and R. P. Agarwal, "Common fixed point theorems for a pair of countably condensing mappings in ordered Banach spaces," Journal of Applied Mathematics and Stochastic Analysis, vol. 16, no. 3, pp. 243-248, 2003. 
[29] Lj. Ćirić, B. Samet, C. Vetro, and M. Abbas, "Fixed point results for weak contractive mappings in ordered $K$-metric spaces," Fixed Point Theory, vol. 13, no. 1, pp. 59-72, 2012.

[30] H. K. Nashine, W. Sintunavarat, and P. Kumam, "Cyclic generalized contractions and fixed point results with applications to an integral equation," Fixed Point Theory and Applications, vol. 2012, article 217, 2012. 


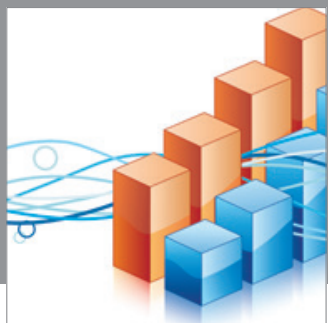

Advances in

Operations Research

mansans

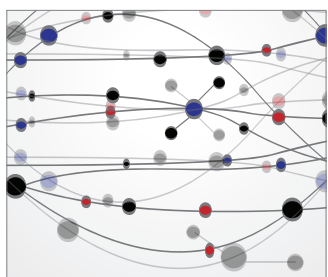

The Scientific World Journal
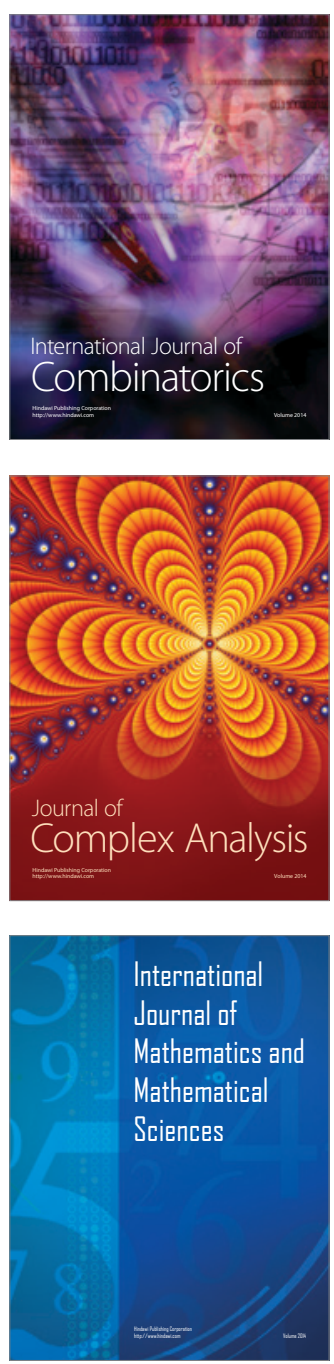
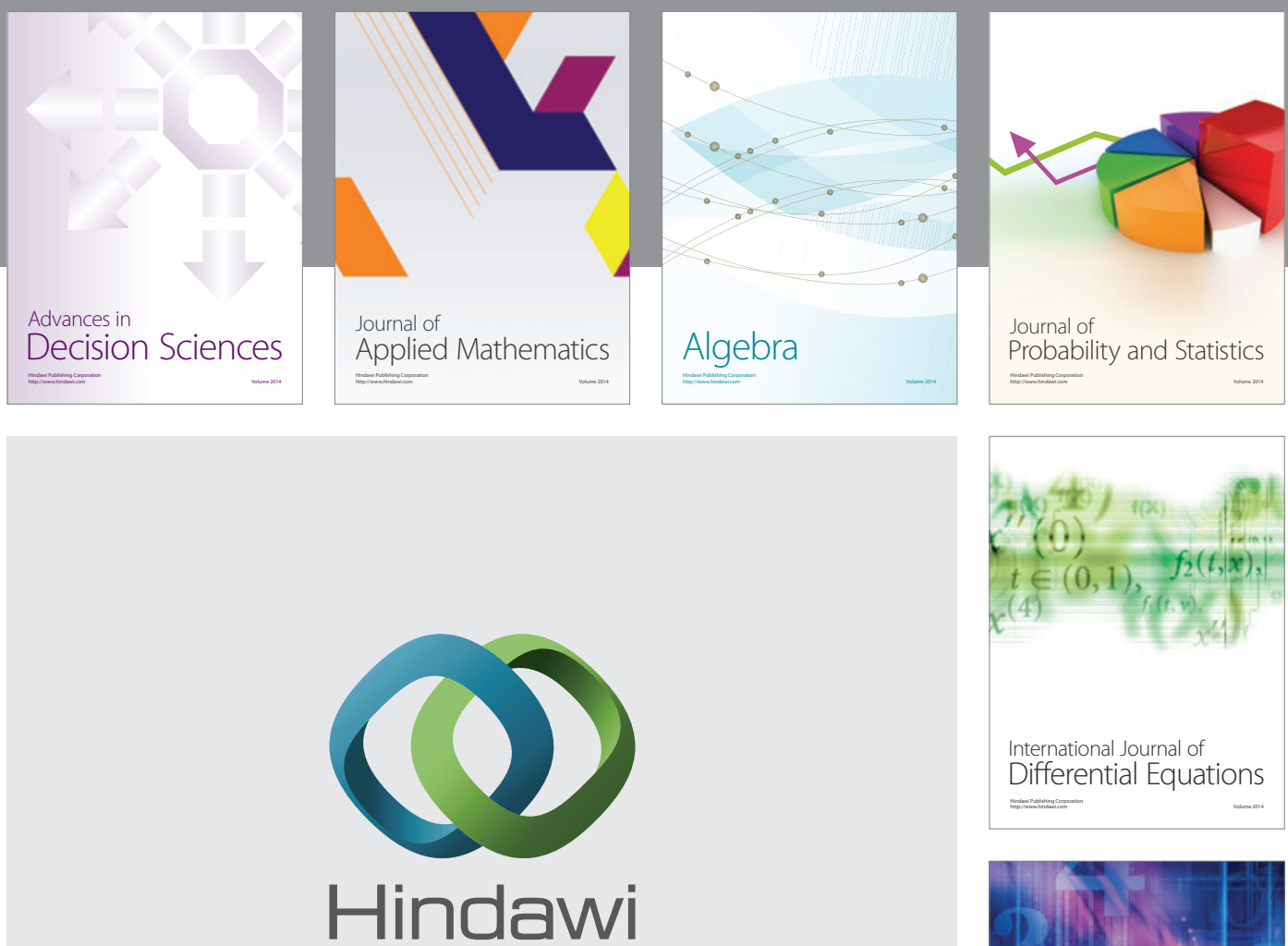

Submit your manuscripts at http://www.hindawi.com
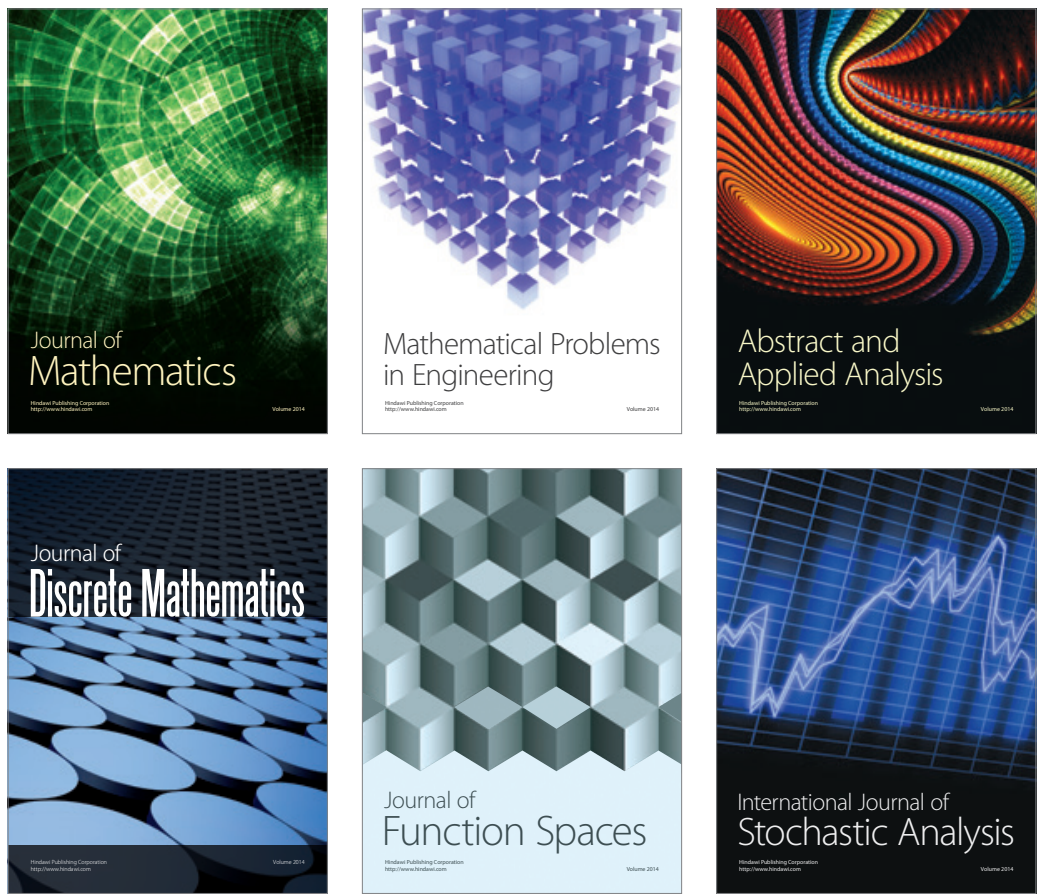

Journal of

Function Spaces

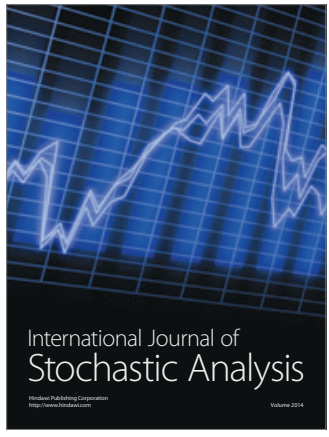

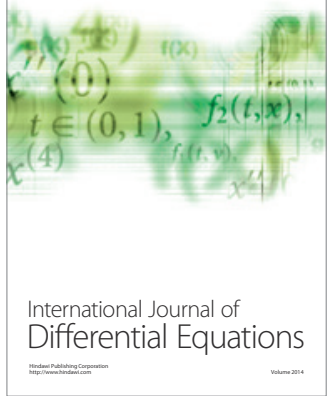
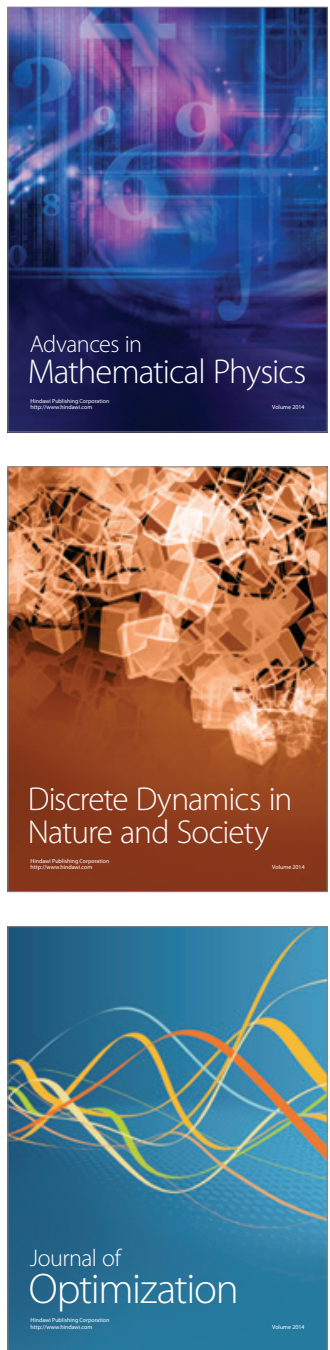